\title{
Deteç̧ão de inovações tecnológicas na evolução da informática educacional no Brasil
}

\author{
Bruno Elias Penteado', Marcelo Fornazin ${ }^{2}$ \\ ${ }^{1}$ Fundação Oswaldo Cruz, Estratégia Fiocruz para a Agenda 2030. Rio de Janeiro, RJ, Brasil. \\ ${ }^{2}$ Fundação Oswaldo Cruz, Escola Nacional de Saúde Pública Sérgio Arouca. Rio de Janeiro, \\ RJ, Brasil.
}

brunopenteado@alumni.usp.br, marcelo.fornazin@ensp.fiocruz.br

\begin{abstract}
Innovations in a knowledge field are usually cyclical and depends on the developed technological infrastructure. In this work we sought to characterize the contributions from informatics in education over the years, providing evidences of innovation cycles in research carried out in Brazil. To that, we applied bibliometric techniques on the main journal of the subject matter in Brazil - the Brazilian Journal of Computers in Education (RBIE) between 1997 and 2021. Three homogeneous research periods were detected, in addition to nine clusters of themes, showing the evolution of these clusters over the periods.
\end{abstract}

Resumo. Inovações em um campo de conhecimento são geralmente cíclicas e dependentes da infraestrutura tecnológica desenvolvida. Neste trabalho buscamos caracterizar as contribuições da informática na educação ao longo dos anos, evidenciando ciclos de inovação nas pesquisas feitas na área no Brasil. Para isso, aplicamos técnicas bibliométricas na principal revista nacional deste assunto - a Revista Brasileira de Informática na Educação (RBIE) entre 1997 e 2021. Três períodos homogêneos de pesquisas foram detectados, além de nove agrupamentos de temas, demonstrando a evolução desses temas ao longo dos períodos.

\section{Introdução}

A informática na educação pode ser considerada como "a inserção do computador no processo de ensino-aprendizagem de conteúdos curriculares de todos os níveis e modalidades de educação" [Valente, 1999]. As primeiras experiências no Brasil ocorreram na década de 1970 dentro de universidades e, desde então, novas tecnologias têm surgido e o barateamento de equipamentos e infraestrutura tem permitido que elas sejam incorporadas à realidade escolar ou mesmo diretamente pelos alunos.

Ao longo do tempo, os computadores vêm sendo incorporados ao contexto escolar, como instrumento de ensino e aprendizagem para diversos assuntos e como objeto de estudo em si, por alunos que desejam aprender conceitos teóricos e práticos 
sobre informática e computação [Posada et al., 2016]. Políticas públicas como o Programa Nacional de Tecnologia Educacional (ProInfo) tiveram papel central na popularização e buscaram disseminar o uso pedagógico das tecnologias de informática e telecomunicações nas escolas públicas de ensino fundamental e médio pertencentes às redes estadual e municipal, por meio do subsídio para a instalação de infraestrutura de informática nas escolas [Elia, 2021].

A produção de trabalhos científicos nesta área de conhecimento também vem aumentando, com a criação de conferências e periódicos tratando deste assunto. Tópicos diferentes têm emergido e se ajustado a partir da evolução dos contextos tecnológicos, sociais, econômicos e políticos do país [Elia, 2021]. Esse crescimento envolve grande complexidade informacional para a compreensão desta área de pesquisa. Conhecer a evolução da área como um todo permite a análise crítica e histórica dos ciclos tecnológicos que surgem e poucos trabalhos abordaram quantitativamente esta questão.

O objetivo deste artigo é o de compreender a dinâmica da pesquisa em informática da educação no Brasil, mostrando sua evolução ao longo dos anos em termos de tecnologias, problemas educacionais atendidos e tendências. Tais transformações podem trazer inovações tecnológicas, novos modelos de negócio e políticas públicas em uma determinada indústria [Tigre \& Noronha, 2012]. Deste modo, colocamos as seguintes questões de pesquisa a serem exploradas a partir da literatura científica da informática na educação no Brasil: QP1) quais são os períodos que apresentam similaridade de temas pesquisados? QP2) quais são os principais temas de pesquisa? QP3) qual a evolução desses temas dentre os períodos detectados?

A partir das respostas a essas perguntas podemos vislumbrar sinteticamente a evolução e a compreensão da área da informática da educação no Brasil a partir dos temas emergentes e sua contextualização histórica.

\section{Referenciais teóricos}

A evolução tecnológica da inovação tem sido estudada em diferentes campos de pesquisa, em especial nas Ciências Econômicas. Trabalhos como o de Utterback \& Suárez [1993] buscam compreender as dinâmicas da mudança tecnológica e apresentar evidências sobre padrões de inovação em produtos e seus processos habilitadores. Os autores dividem as grandes inovações tecnológicas em cinco períodos: revolução industrial, a era do vapor, a era da eletricidade, a produção em massa, as tecnologias de informação e comunicação, destacando os potenciais técnicos, o timing e outras circunstâncias. Tigre \& Noronha [2012] investigaram mudanças estruturais ocorridas na indústria de TIC das cinco décadas mais recentes até então, analisando o surgimento de empresas-paradigma e seus respectivos modelos de negócio. Os autores identificaram cinco mudanças tecnológicas que trouxeram novos paradigmas emergentes: os mainframes, os minicomputadores, os microcomputadores, a Internet e a computação em nuvem. Lente et al. [2013] buscaram modelar ondas de inovação tecnológica conforme atenção dada pelas mídias combinado com expectativas crescentes nas possibilidades tecnológicas, num fenômeno conhecido 
como hype, em que é estudada a dinâmica das expectativas nos processos de inovação e que podem levar a grandes realizações ou decepções. Foram encontradas ondas de diferentes padrões de formatos, modulados pelas expectativas em diferentes níveis, desde o micro (projetos individuais) até o macro (mudanças sociais).

Os modelos citados analisaram a evolução da indústria, em seus produtos, processos e serviços. Bramoullé \& Saint-Paul [2010] analisaram o contexto acadêmico, investigando ciclos de pesquisa, demostrando dois tipos de ondas: i) em que novos campos são constantemente inventados e convergem para um estado estável e ii) ciclos em que os períodos de inovação se alternam com períodos de exploração. Os resultados se mostraram em linha com os pressupostos teóricos de Kuhn [1970], em que a ciência evolui se alternando entre períodos de ciência incremental, baseada em contribuições passadas e de revoluções, que trazem novos parâmetros de referência. Assume-se a ocorrência de alguma forma de retorno decrescente, o que garante que, se a exploração durar o suficiente, a inovação radical pode valer a pena. Ciclos emergem devido ao fato de monopólios dos pioneiros serem temporários [Matsuyama, 1999] e da sua estrutura organizacional [Garicano \& Rossi-Hansberg, 2012].

Cada um desses autores adota diferentes granularidades sobre as transformações cíclicas geradas pelas inovações tecnológicas, como diferentes indústrias, as TICs. Os autores concluem que tais ondas ocorrem mesmo em diferentes tipos de indústria, sendo sensíveis ao contexto do potencial da tecnologia e de seu momento, apresentando modelos que convergem para a detecção de ondas de inovação tecnológica. Tais estudos adotaram metodologias qualitativas, investigando estudos de caso, a partir de amostragem teórica, buscando generalização com diferentes sujeitos dentro do escopo determinado. Neste trabalho, seguindo as suposições teóricas da existência de ondas de inovação tecnológica, buscamos identificar quais foram essas ondas e como elas se caracterizaram.

Nos últimos anos, o campo da ciência de informação tem evoluído para capturar o estudo de produções intelectuais em diferentes formatos: publicações acadêmicas, redes sociais, patentes, dentre outras fontes [Curty \& Delbianco, 2020]. Um desses ramos é a bibliometria, responsável por elaborar indicadores e estudar fenômenos da produção na literatura acadêmica, tendo como unidades de análise: os autores, os trabalhos e seus metadados, as referências, o impacto, os veículos de publicação [Haddow, 2018]. Ferramentas de software têm sido desenvolvidas para dar suporte a este corpo de conhecimento, implementando diferentes indicadores e visualizações. Uma vantagem desta abordagem diz respeito a oferecer análises empíricas em torno destas unidades de análise e quantificar padrões e tendências presentes nas características da literatura de interesse [Haddow, 2018]. É uma abordagem complementar a outras formas de mapeamento da literatura cientifica, como as revisões sistemáticas, pois fornece indicadores estatísticos que descrevem a estrutura de conhecimento e o impacto de artigos, autores ou revistas em um campo de pesquisa.

A partir destas características, adotamos a bibliometria como forma de capturar possíveis ondas de inovação em informática na educação a partir de dados empíricos da 
literatura científica. Para isso, adotamos técnicas que buscam identificar e caracterizar tais ondas de inovação conforme ocorridas ao longo dos anos. Esta abordagem se diferencia de outras presentes na literatura ao analisar quantitativamente a produção cientifica da área como um todo, buscando maior generalização dos resultados.

\section{Trabalhos relacionados}

A história e a evolução da área de Informática na Educação têm sido narradas por diversos autores, a partir de diferentes métodos. Moraes [1997] inaugura a da Revista Brasileira de Informática na Educação (RBIE) trazendo uma perspectiva histórica e cronológica de acontecimentos que possibilitaram o nascimento da informática educacional no Brasil, seus principais projetos de fomento governamental e os resultados parciais. Valente [1999] discorre sobre as origens, as influências internacionais, projetos e políticas públicas até aquele momento. Traz referências às primeiras iniciativas de inserção de computadores para fins educacionais ainda na década de 1970 e discute a finalidade da informática na educação e a importância da formação dos professores para realizar seu potencial.

Elia [2021], por meio de narrativa autobiográfica de um agente presente desde as primeiras iniciativas no Brasil, ressalta a importância das políticas públicas - em especial as duas edições do ProInfo - como catalisador da adoção da tecnológica à prática pedagógica nas escolas públicas brasileiras. $\mathrm{O}$ autor também argumenta sobre a influência de outros agentes nesse contexto (academia, empresas, escolas e comunidade escolar) e o contexto político-social-cultural-econômico que traz a acelerada adoção das tecnologias da informação em todos os contextos que permeiam nossas vidas, incluindo a educação. Nesse recorte, traz algumas das inovações tecnológicas que impactaram essa transformação, em linha com as apresentadas por Tigre \& Noronha [2012].

O trabalho mais similar ao proposto neste artigo é o de Posada et al. [2016], onde os autores realizaram também uma análise bibliométrica dos dados científicos dos artigos publicados em revista e conferências brasileiras, ressaltando a rede de autores, expressões e períodos. Além disso, os autores utilizam dados de outras fontes relacionadas (SBIE, WIE e CBIE) nos períodos de 1997 a 2014. No entanto, o presente artigo se diferencia deste outro nos seguintes aspectos: i) utiliza palavras-chave como fonte de informação, já que elas são consideradas os elementos básicos da representação de conceitos de conhecimento, denotando a intencionalidade do autor e têm sido usadas para revelar domínios de pesquisa [Chen \& Xiao, 2016]. O uso de títulos e resumos tem sua qualidade dependente do algoritmo que extrais expressões desses textos livres. Em Posada et al. (2016) foi feita a contagem de n-gramas, sem detalhar atividades de pós-processamento ou de remoção de ruídos; ii) o uso de agrupamentos (clusters) para sintetizar estudos relacionados, oferecendo uma visão mais compacta e robusta em torno dos tópicos pesquisados, uma vez que os dados trazem muito ruído ( $c f$. Figura 1, com distribuição de frequência das palavras-chave); iii) uso de outros artefatos visuais para ilustrar a evolução do campo. As nuvens de tag utilizadas em Posada et al. (2016) apresentam limitações, 
como por exemplo a predominância visual dos termos mais comuns sem capturar estruturas subjacentes, conforme literatura sobre o tema [Khusro et al., 2021; Sinclair \& Cardew-Hall, 2008]; iv) o uso de detecção automática de períodos pela similaridade dos artigos em anos consecutivos, o que torna possível a descoberta de ciclos de inovação nas pesquisas, conforme aporte teórico adotado.

Este artigo estende a literatura ao fornecer uma análise empírica sobre a produção científica da informática educacional no Brasil, fornecendo evidências quantitativas sobre as diferentes ondas de inovação tecnológica ocorridas nos últimos 25 anos, apoiadas pelas técnicas bibliométricas de análise da informação. Esta abordagem foi aplicada, por exemplo, no estudo da evolução das tecnologias na área da saúde, identificando diferentes ondas e suas inovações tecnológicas [Fornazin et al., 2021; Penteado et al, 2021].

\section{Metodologia}

Para compreender a evolução dos temas de informática na educação no Brasil foi criado um script de raspagem de dados que recuperou as palavras-chave, anos, títulos, autores e resumos de todos os artigos publicados e disponíveis na página da Revista Brasileira de Informática na Educação ${ }^{1}$ em julho de 2021, entre 1997 e 2021, num total de 548 artigos e 874 palavras-chave distintas. Aqui, assumimos esta revista como um proxy para o desenvolvimento da informática na educação no Brasil, sendo o periódico mais bem conceituado pela Qualis-CAPES nesta área. Dentre o total de artigos recuperados, 39 não apresentaram palavras-chave $(6,5 \%)$, sendo editoriais ou resumos publicados nos primeiros volumes, sem apresentar esses metadados.

As Figuras 1 e 2 ilustram a distribuição anual dos artigos e as frequências das palavras-chave. Podemos notar que existe uma quantidade representativa a cada ano, justificando a revista como um proxy para a produção na área. Nos primeiros anos da RBIE foram publicados artigos com resumos estendidos e que não foram considerados no processamento. Além disso, pode-se notar uma distribuição paretiana em relação à frequência das palavras-chave, em que poucas palavras se mostraram predominantes e uma cauda longa é observada com os termos menos frequentes (Figura 2).

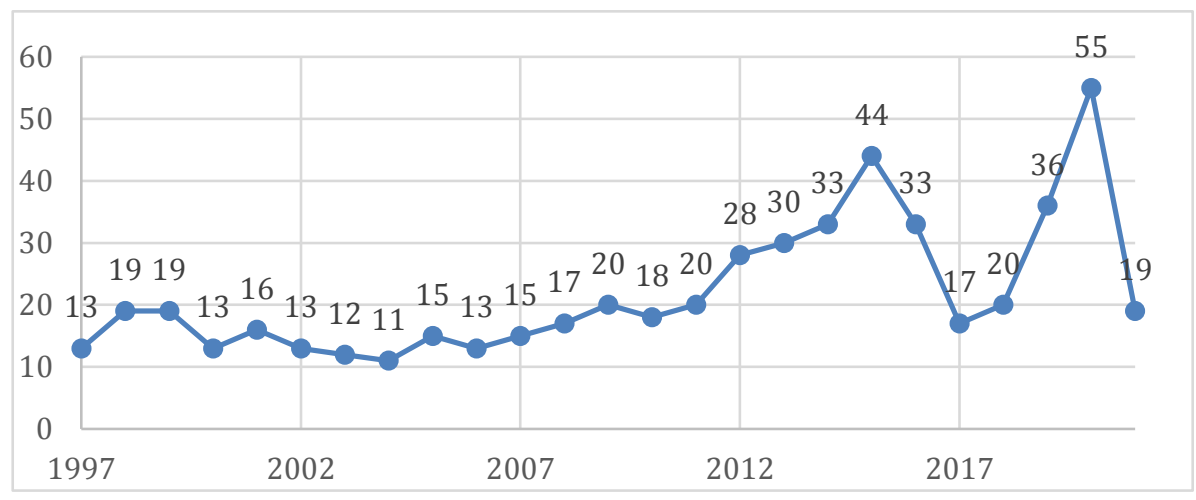

Figura 1. Número de artigos por ano publicados na RBIE.

\footnotetext{
${ }^{1}$ https://www.br-ie.org/pub/index.php/rbie/issue/archive
} 


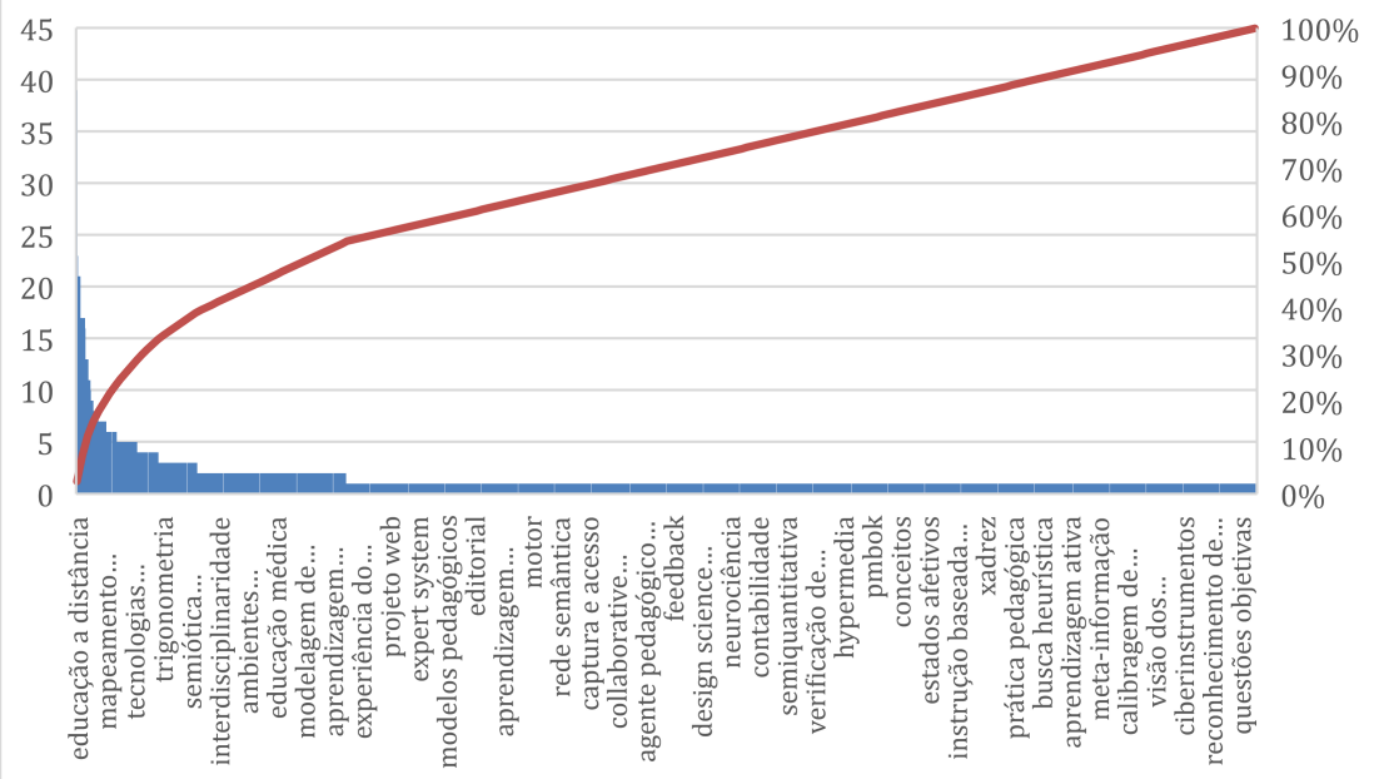

Figura 2. Distribuição da ocorrência das palavras-chave encontradas nos artigos da RBIE.

Muitos destes termos apresentam variações linguísticas de um mesmo conceito, tendo sido normalizadas em relação a número, locuções, abreviações e língua ${ }^{2}$. Em seguida, apenas os termos com no mínimo 5 ocorrências foram mantidos, de modo a eliminar ruídos com palavras-chave muito esparsas. Com isso, o número de termos normalizado foi de 45, cobrindo 83,6\% dos artigos com palavras-chave. Foi utilizado o software CorText (www.cortext.net) para fazer a normalização e realizar as análises das questões de pesquisa descritas a seguir.

Para a QP1 foi utilizada a funcionalidade de detecção de períodos, que cria um vetor de ocorrências de palavras-chave (bag-of-words) para cada ano e calcula, para cada par de anos, seu grau de similaridade. Foi usada a estimativa automática de quantos agrupamentos de anos buscar, no qual o algoritmo escolhe o valor ótimo de acordo com critério estatístico gap, conforme descrito em Tbishirani et al. (2001).

Para a QP2, aplicamos um mapeamento de redes de co-ocorrência de palavraschave - método comumente utilizado para visualizar uma estrutura de conhecimento de um campo de pesquisa [Su \& Lee, 2010]. Para cada par de palavras-chave em um artigo é criada uma aresta que liga os dois nós que representam as palavras-chave. Para diminuir a influência de ruídos, limitamos aos termos normalizados com no mínimo 5 ocorrências no corpus processado. Os agrupamentos foram calculados usando algoritmo de Louvain para detecção de comunidades em grafos e cada artigo foi atribuído ao agrupamento mais próximo.

Para a QP3, utilizamos diagrama de Sankey para visualização da evolução, representando a proporção de artigos de cada agrupamento para cada um dos períodos

\footnotetext{
2 Por exemplo: ambiente virtual de aprendizagem, ambientes virtuais de aprendizagem, AVA, virtual learning environment, LMS.
} 
detectados, ordenados pela sua predominância. Com isso, é possível identificarmos visualmente os ciclos de inovação nas pesquisas acadêmicas ao longo dos períodos.

\section{Resultados}

A Figura 3 ilustra os períodos detectados automaticamente pelo algoritmo de semelhança de vetores de palavras-chave. No triângulo inferior são apresentados os graus de dissimilaridade entre cada par de anos individualmente. Quanto mais branca a célula da interseção entre dois anos, mais diferentes elas são; pelo outro lado, quanto mais verdes, mais similares (como na diagonal principal, em que os anos são comparados a si mesmos). O triângulo superior demonstra o agrupamento dessas dissimilaridades. Forma detectados três períodos: 1997-2004, 2005-2011 e 2012-2021, sendo este último mais coeso que os anteriores.

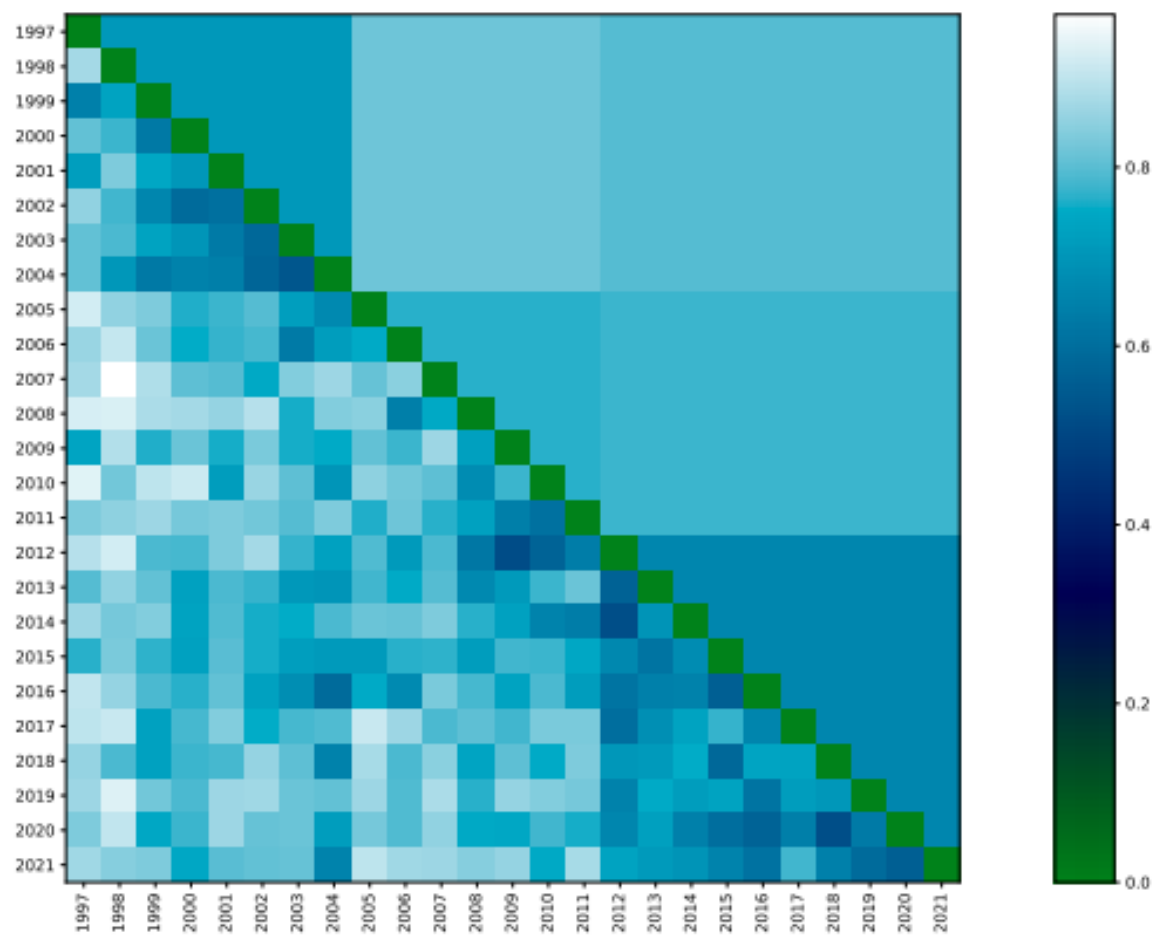

Figura 3. Detecção dos períodos por semelhanças de palavras-chave a cada ano.

Os agrupamentos das coocorrências detectados pelo algoritmo estão ilustrados na Figura 4, preservando topologicamente a similaridade entre eles. Dos nove agrupamentos encontrados, apenas dois apresentaram grande sobreposição: educação \& aprendizagem e objetos de aprendizagem \& formação de professores, com os termos educação e informática na educação sendo parte de ambos, porém alocados em agrupamentos diferentes conforme critério de maior proximidade do algoritmo. A Tabela 1 descreve quais termos foram alocados a quais agrupamentos. 


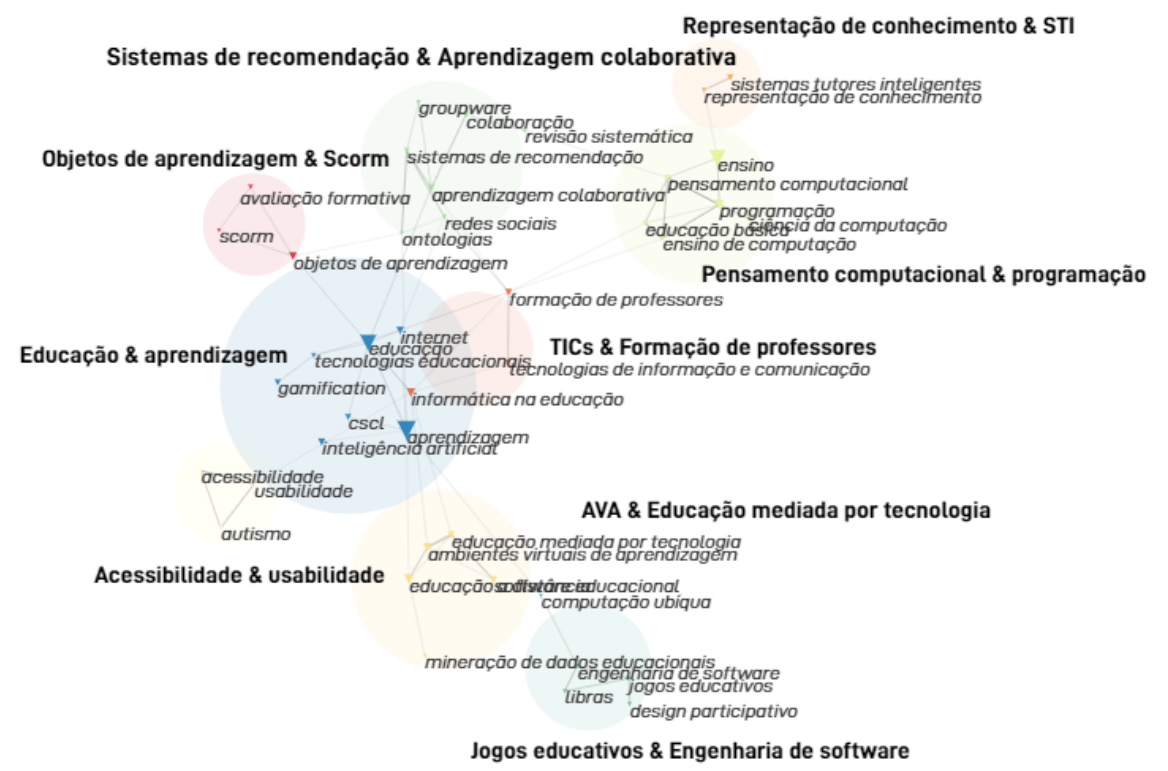

Figura 3. Agrupamentos detectados e sua disposição topográfica.

Tabela 1. Agrupamentos detectados e seus termos estruturantes.

\begin{tabular}{|c|c|}
\hline Agrupamento & Termos \\
\hline Educação \& aprendizagem & $\begin{array}{l}\text { Educação, aprendizagem, Internet, CSCL, inteligência artificial, } \\
\text { gamification, tecnologias educacionais }\end{array}$ \\
\hline $\begin{array}{l}\text { Sistemas de recomendação } \& \\
\text { Aprendizagem colaborativa }\end{array}$ & $\begin{array}{l}\text { Colaboração, groupware, revisão sistemática, sistemas de } \\
\text { recomendação, aprendizagem colaborativa, redes sociais, } \\
\text { ontologias }\end{array}$ \\
\hline $\begin{array}{lll}\begin{array}{l}\text { Pensamento } \\
\text { programação }\end{array} & \text { computacional } & \& \\
\end{array}$ & $\begin{array}{l}\text { Ensino, pensamento computacional, ciência da computação, } \\
\text { educação básica, ensino de computação, programação }\end{array}$ \\
\hline Acessibilidade \& usabilidade & Acessibilidade, usabilidade, autismo \\
\hline $\begin{array}{l}\text { Representação de conhecimento \& } \\
\text { Sistemas tutores inteligentes }\end{array}$ & Representaç \\
\hline $\begin{array}{l}\text { Ambientes virtuais de aprendizagem } \\
\text { \& Educação mediada por tecnologia }\end{array}$ & $\begin{array}{l}\text { Ambientes virtuais de aprendizagem, educação mediada por } \\
\text { tecnologia, software educacional, educação a distância, } \\
\text { mineração de dados }\end{array}$ \\
\hline $\begin{array}{l}\text { Jogos educativos \& Engenharia de } \\
\text { software }\end{array}$ & $\begin{array}{l}\text { Jogos educativos, engenharia de software, Libras, design } \\
\text { participativo }\end{array}$ \\
\hline $\begin{array}{llll}\text { Objetos } & \text { de } & \text { aprendizagem } & \& \\
\text { SCORM } & & & \\
\end{array}$ & Objetos de aprendizagem, SCORM, avaliação formativa \\
\hline TICs \& Formação de professores & $\begin{array}{llll}\begin{array}{l}\text { Formação de professores, tecnologias } \\
\text { comunicação, informática na educação }\end{array} & \text { informação e } \\
\end{array}$ \\
\hline
\end{tabular}


A Figura 5 ilustra a evolução de cada tema (agrupamento) detectado ao longo dos três períodos detectados automaticamente.

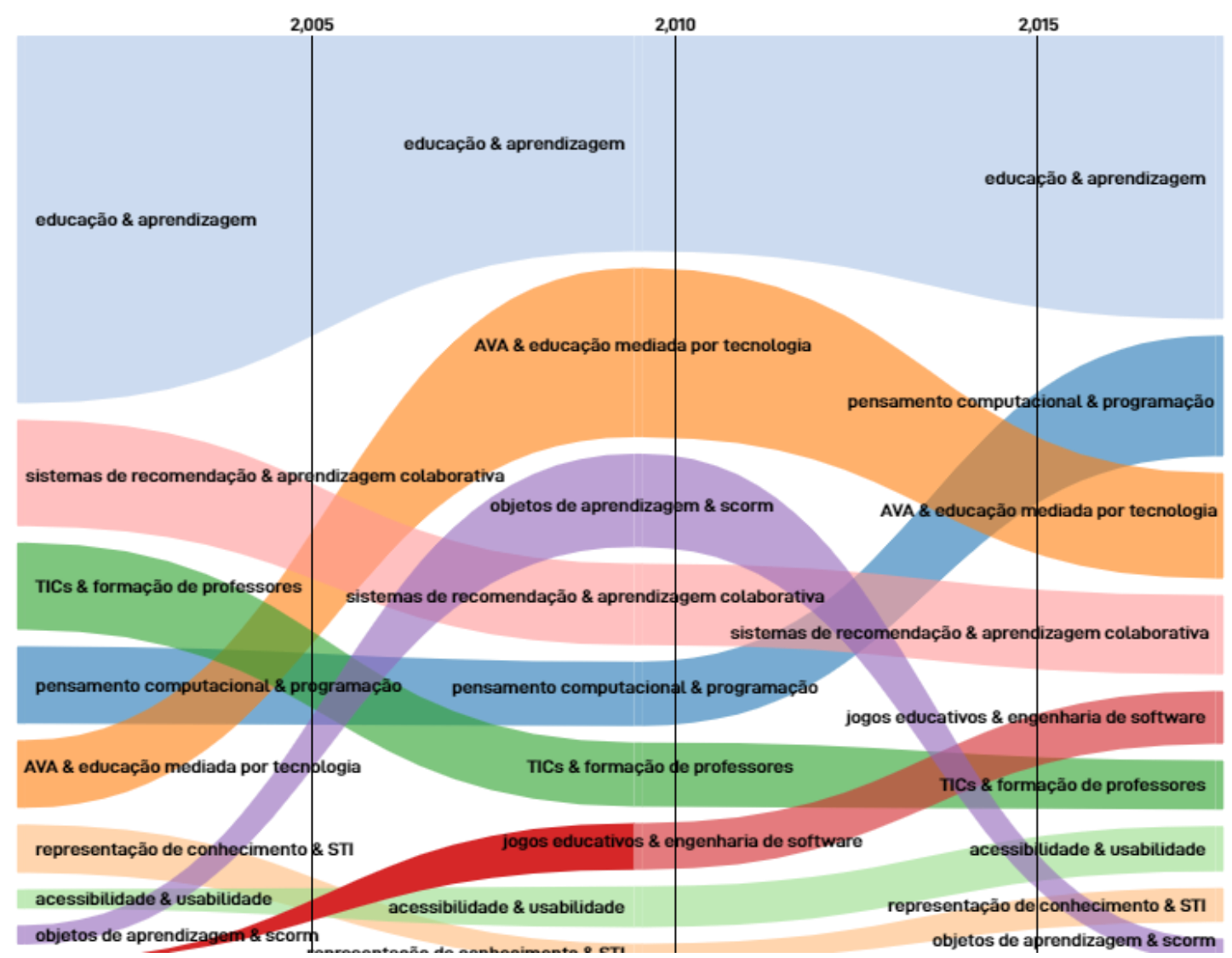

Figura 5. Evolução de cada agrupamento ao longo dos períodos detectado.

\section{Discussão}

Por meio das análises bibliométricas foi possível determinar períodos homogêneos quanto a estrutura de conhecimento subjacente. Foram encontrados nove agrupamentos temáticos relativamente disjuntos e coerentes. $\mathrm{O}$ agrupamento mais frequente em todos os períodos traz termos genéricos (educação, aprendizagem, tecnologias educacionais) bem como termos mais específicos como CSCL, inteligência artificial e gamification. $\mathrm{O}$ agrupamento de aprendizagem colaborativa traz termos relacionados ao assunto (colaboração, groupware, aprendizagem colaborativa, redes sociais) bem como outros mais genéricos (revisão sistemática, ontologias), sugerindo um sobreajuste a artigos que tenham usado essas coocorrências, já que o limite de ocorrências foi mantido baixo (5). O mesmo acontece a outros agrupamentos. Porém, argumentamos que os agrupamentos, de forma geral, demonstram conceitos coerentes entre si, variando em termos heterogêneos ou genéricos e isolados.

Dentro dos ciclos de inovação, alguns padrões foram encontrados. Os resultados do agrupamento de objetos de aprendizagem mostram um ciclo decrescente, depois de ser altamente pesquisado no segundo período. Teve seu pico com tópicos como metodologias de desenvolvimento, padronização SCORM e agrupamento de objetos 
semelhantes. No último período, recomendação e sequenciamento de objetos. A onda de ambiente virtuais de aprendizagem (AVA) se mostrou estável, tendo um grande crescimento a partir do segundo período e se caindo pouco no terceiro. Inicialmente, este agrupamento traz a análise de ferramentas e práticas (por ex. laboratórios, cadernos digitais, realidade aumentada) em AVAs. A mineração de dados educacionais aplicado a esse tipo de ambiente (por ex: predição de evasão e reprovação, atividades em MOOC) foi o destaque deste agrupamento nos últimos anos. Já a onda de pensamento computacional demonstrou um grande salto no último período, demostrando uma tendência para os próximos anos. Artigos que abordaram o ensino de computação na educação básica, alinhados a diretrizes da Base Nacional Comum Curricular (BNCC) e o ensino de programação em cursos de diferentes níveis caracterizaram este agrupamento. No último ano houve um maior número de artigos relacionados a ensino remoto devido à Covid-19, mas que não foi detectado pelo algoritmo nesta base de artigos, por estar presente em diferentes agrupamentos simultaneamente.

O presente artigo traz algumas limitações, que deverão ser tratadas em trabalhos futuros. O uso das palavras-chave limitou o alcance da base de artigos analisados. Artigos extraídos de outras fontes, como os utilizados por Posada et al. (2016), não apresentam esta informação nos metadados onde as informações foram coletadas. Possivelmente, um número maior de artigos traria uma maior generalização dos agrupamentos, tornando-os mais coerentes e menos dependentes de um número pequeno de artigos sobre o tema. De qualquer maneira, acreditamos que o proxy utilizado (a RBIE) é representativo desse universo e traz uma avaliação mais criteriosa que as conferências correlatas. Devido à limitação do número de páginas deste artigo, não foi possível avaliar qualitativamente trabalhos relevantes em cada período, o que seria desejável para uma análise mais bem qualificada sobre a caracterização dos períodos e agrupamentos. Do mesmo modo, a exploração das causas de cada onda deve ser aprofundada para melhor buscar compreender quais foram os fatores que mais impactaram a evolução deste campo no Brasil.

\section{Referências}

Bramoullé, Y.; Saint-Paul, G. (2010). Research cycles. Journal of Economic Theory, 145, p. 1890-1920. DOI: 10.1016/j.jet.2010.02.004.

Chen, G., and Xiao, L. 2016. Selecting publication keywords for domain analysis in bibliometrics: A comparison of three methods, Journal of Informetrics (10), pp. 212223.

Curty, R. G.; Delbianco, N. R. (2020). As diferentes metrias dos estudos métricos da informação: evolução epistemológica, inter-relações e representações. Encontros Bibli: revista eletrônica de biblioteconomia e ciência da informação, Florianópolis, v. 25, p. 01-21, 2020. DOI: 10.5007/1518-2924.2020.e74593.

Elia, M. F. (2021). A História da Informática na Educação no Brasil: uma narrativa em construção. In: SANTOS, Edméa O.; SAMPAIO, Fábio F.; PIMENTEL, Mariano (Org.). Informática na Educação: sociedade e políticas. Porto Alegre: Sociedade Brasileira de Computação, 2021. (Série Informática na Educação CEIE-SBC, v.4) 
Disponível em: https://ieducacao.ceie-br.org/historiainformaticaeducacao.

Fornazin, M.; Penteado, B. E.; Castro, L. C.; Silva, S. L. F. C. (2021). From Medical Informatics to Digital Health: A Bibliometric Analysis of the Research Field. Americas' Conference on Information Systems, número 18. Disponível em: https://aisel.aisnet.org/amcis2021/healthcare_it/sig_health/18.

Garicano, L.; Rossi-Hansberg, E. (2012). Organizing growth, Journal of Economic Theory, v. 147 (2), p. 623-656. DOI:10.1016/j.jet.2009.11.007.

Haddow, G. (2018). Bibliometric research. Research methods: Information, systems, and contexts. In K. Williamson, G. Johanson (Ed.). 2a edição, Elsevier, p. 241-266.

Khusro, S.; Jabeen, F.; Khan, A. (2021). Tag Clouds: Past, Present and Future. Proceedings of the National Academy of Science, India Section. A Phys. Sci. 91, 369381. DOI: $10.1007 / \mathrm{s} 40010-018-0571-\mathrm{x}$.

Kuhn, T. (1970). The Structure of Scientific Revolutions, Chicago University Press, Chicago, IL.

Lente, H.; Spitters, C.; Peine, A. (2013). Comparing technological hype cycles: Towards a theory. Technological Forecasting and Social Change, v 80 (8), p. 1615-1628. DOI: 10.1016/j.techfore.2012.12.004.

Matsuyama, K. (1999). Growing through cycles, Econometrica 67 (1999) 335-347.

Penteado B.E., Fornazin M., Castro L. (2021) The Evolution of Artificial Intelligence in Medical Informatics: A Bibliometric Analysis. In: Marreiros G., Melo F.S., Lau N., Lopes Cardoso H., Reis L.P. (eds) Progress in Artificial Intelligence. EPIA 2021. Lecture Notes in Computer Science, vol 12981. Springer, Cham. https://doi.org/10.1007/978-3-030-86230-5_10.

Posada, J. E. G.; Buchdid, S. B.; Baranauskas, M. C. C. (2016). A informática na educação: o que revelam os trabalhos publicados no Brasil. Revista Brasileira de Informática na Educação, v 24 (1), DOI: 10.5753/RBIE.2016.24.01.142.

Sinclair, J., \& Cardew-Hall, M. (2008). The folksonomy tag cloud: when is it useful? Journal of Information Science, 34(1), 15-29. DOI: 10.1177/0165551506078083.

Su H., Lee P. Mapping knowledge structure by keyword co-occurrence: a first look at journal papers in Technology Foresight. Scientometrics, 85, pp. 65-70. (2010). DOI: $10.1007 / \mathrm{s} 11192-010-0259-8$

Tibshirani, R., Walther, G., and Hastie, T. 2001. "Estimating the number of clusters in a data set via the gap statistic," Journal of the Royal Statistical Society (63:2), pp. 411423.

Tigre, P. B.; Noronha, V. B. (2012). Do mainframe à nuvem: inovações, estrutura industrial e modelos de negócios nas tecnologias da informação e comunicação. Revista de Administração (São Paulo), v. 48 (1), p. 114-127.

Utterback, J.; Suárez, F. (1993). Innovation, competition and industry structure. Research Policy, Elsevier, London, v. 22 (1), p.1-21.

Valente, J. A. (1999). Informática na educação no Brasil: análise e contextualização histórica. O computador na sociedade do conhecimento. Campinas: UNICAMP/NIED, p. 1-13. 